\title{
Carboidratos na Dieta Pré-Inicial de Frangos de Corte ${ }^{1}$
}

\section{Flavio Alves Longo ${ }^{2}$, José Fernando Machado Menten ${ }^{3}$, Adriana Ayres Pedroso², Adriana Nogueira Figueiredo ${ }^{2}$, Aline M. Calil Racanicci², Juliano Benedito Gaiotto ${ }^{2}$, José Otávio Berti Sorbara ${ }^{2}$}

\begin{abstract}
RESUMO - Objetivou-se, com este estudo, determinar a energia metabolizável aparente corrigida (EMAn) de ingredientes como fonte de carboidrato para frangos de corte de 1 a 7 dias de idade, bem como avaliar a utilização destes diferentes ingredientes em dietas préiniciais sobre o desenvolvimento inicial de órgãos do trato gastrintestinal (TGI) e desempenho das aves. No Experimento 1, foi utilizado o método de coleta total das excretas com 220 pintos de 4 a 7 dias de idade, alojados em baterias em delineamento inteiramente casualizado com cinco tratamentos (uma dieta-referência e quatro dietas com inclusão de $20 \%$ do ingrediente-teste - amido de milho [AMI], amido de mandioca [AMA], glicose [GLI] e sacarose [SAC]) e quatro repetições de 11 aves. No Experimento 2, foram utilizados 520 pintos de corte machos de um dia de idade, distribuídos em delineamento inteiramente casualizado, com cinco tratamentos e quatro repetições de 26 aves. Os tratamentos foram aplicados de 1 a 7 dias e, no período subseqüente, as aves tiveram acesso a uma dieta basal padrão. Os tratamentos consistiram em: 1 - dieta de milho e farelo de soja, 2 - dieta de milho e farelo de soja + AMI, 3 - dieta de milho e farelo de soja + AMA, 4 - dieta de milho e farelo de soja + GLI, 5 - dieta de milho e farelo de soja + SAC. O valor da EMAn determinado para frangos de corte na fase pré-inicial foi de 3.269, 3.690, 3.427 e $3.524 \mathrm{kcal} / \mathrm{kg}$ para AMI, AMA, GLI e SAC, respectivamente. Observou-se que o desenvolvimento do TGI, de maneira geral, não foi influenciado pelos diferentes tratamentos, mas o consumo de ração das aves foi aumentado com o fornecimento de carboidrato de maior disponibilidade na dieta pré-inicial, contribuindo para aumento no peso vivo das aves.
\end{abstract}

Palavras-chave: carboidratos, desempenho, dieta pré-inicial, energia metabolizável, frangos de corte e trato gastrintestinal

\section{Carbohydrates in the Diets of Newly Hatched Chicks}

\begin{abstract}
This work was conducted to determine the N-corrected apparent metabolizable energy (EMAn) of different carbohydrate sources for broilers from 1 to 7 days, and to evaluate the utilization of these ingredients in the diet of newly hatched broilers based and on live performance and on the gastrointestinal tract (TGI) development. Total excreta collection method was used in trial one, with 220 male chicks from 4 to 7 days, allocated to batteries in a completely randomized design with five treatments (a reference diet and four diets with inclusion of $20 \%$ of the test ingredients) and four replicates of 11 birds. The ingredients evaluated were corn starch (AMI), cassava starch (AMA), glucose (GLI) and sucrose (SAC). In Trial 2, 520 day-old male chicks were allotted to a completely randomized design with five treatments and four replicates of 26 birds. The birds received the treatments $(1-$ corn and soybean meal diet, 2 - corn and soybean meal diet + AMI, 3 - corn and soybean meal diet + AMA, 4 - corn and soybean meal diet + GLI, 5 - corn and soybean meal diet + SAC) only from 1 to 7 days. From 8 to 21 days, all the birds received a standard diet. The EMAn values of the ingredients determined for broilers in the first week were 3,269; 3,690; 3,427 and 3,524 kcal/kg for AMI, AMA, GLI and SAC, respectively. In general, the results showed that the treatments did not affect the TGI development, but feed intake was improved by the more available carbohydrate sources in the newly hatched chicks diets, contributing to a higher liveweight of the birds.
\end{abstract}

Key Words: broilers, carbohydrates, gastrointestinal tract, metabolizable energy, newly hatched chick diets, performance

\section{Introdução}

A taxa de crescimento inicial da ave pode ser afetada pela quantidade de saco vitelino residual, qualidade e quantidade de alimento e água, nível de enzimas pancreáticas eintestinais, área de superfície dotratogastrintestinal (TGI), transportadores de nutrientes e, principalmente, pela digestibilidade dos nutrientes (Dibner, 1996).
O maior desafio da nutrição pré-inicial em aves, considerando-se a digestibilidade dos nutrientes, parece ser a mudança no tipo de nutrientes fornecidos, que passam de exclusivamente proteínas e gorduras do saco vitelino para uma dieta composta predominantemente por carboidratos.

Dietas com alto teor de carboidrato fornecidas logo após o nascimento das aves promoveram

\footnotetext{
1 Parte da tese de doutorado do primeiro autor desenvolvida na ESALQ/USP. Projeto financiado pela FAPESP

${ }^{2}$ Estudantes de Pós-Graduação em Ciência Animal e Pastagens, ESALQ/USP, Piracicaba, SP.

3 Professor do Depto. de Zootecnia, ESALQ/USP, Piracicaba, SP (jfmmente@esalq.usp.br).
} 
maior concentração de glicose no sangue e queda na atividade da glicose-6-fosfatase, o que indica redução da gliconeogênese (Donaldson \& Christensen, 1991). Esse decréscimo na necessidade de gliconeogênese, para atender à exigência em energia e manter os níveis de glicose circulante a partir de reservas protéicas do saco vitelino e corporais, contribui com maior disponibilidade dessa proteína para formação do tecido corporal e desenvolvimento da ave neonata. Entretanto, o TGI encontra-se imaturo durante a fase embrionária e, após a eclosão, passa a ter grande representatividade no crescimento das aves. Nos primeiros dias de vida, os segmentos do TGI sofrem sensíveis alterações morfológicas e fisiológicas que preparam a ave para o consumo e a utilização de alimentos (Mozdziak et al., 2002; Iji et al., 2001a; Noy et al., 2001; Murakami et al., 1992). Essa imaturidade do TGI de aves na fase préinicial reduz a capacidade de utilização dos nutrientes, o que parece acarretar decréscimo dos valores de energia metabolizável (EM) do alimento. Resultados de literatura mostraram que os valores de EM de alimentos são menores, principalmente entre 4 e 7 dias de idade dos pintos (Sulistiyanto et al., 1999).

$\mathrm{O}$ aproveitamento de um nutriente pela ave depende da digestão e absorção de macromoléculas, o que requer hidrólise enzimática. A disponibilidade da energia proveniente da metabolização de carboidratos, independentemente se proveniente de fonte purificada ou ingredientes ricos nesse nutriente-menor no primeiro dia de idade das aves - é altamente dependente da idade, em decorrência do perfil de atividade da amilase no pâncreas e no intestino delgado (Akiba \& Murakami, 1995).

A digestão de carboidratos ocorre tanto no lúmen como na membrana borda em escova dos enterócitos do intestino delgado. A enzima amilase pancreática hidrolisa o amido da dieta a oligossacarídeos (maltose, maltotriose) e $\alpha$-dextrinas. A digestão final desses produtos, bem como a hidrólise dos dissacarídeos (sacarose, trealose e lactose) ocorre na membrana dos enterócitos pelas dissacaridases (Chotinsky et al., 2001). As dissacaridases são enzimas ligadas à membrana intestinal e qualquer alteração na superfície onde estas enzimas estão ligadas pode alterar as suas atividades. Sell et al. (1991) observaram que aves alimentadas com dietas ricas em carboidratos apresentaram maiores atividades de dissacaridases. Anteriormente, Siddons (1972) constatou que dietas sem carboidratos levaram à redução da atividade das dissacaridases, comprovando que a concentração de carboidrato da dieta promove mudanças na atividade das dissacaridases intestinais. Moran Jr. (1985) indicou que a digestão e a absorção de carboidratos em aves não são fixas, mas são altamente adaptáveis de acordo com sua presença na dieta.

A capacidade de digestão de carboidratos, como o amido, é observada logo após a eclosão, sendo a amilase pancreática encontrada em aves já no 18 o dia de incubação, com sua máxima atividade específica ocorrendo quatro dias após a eclosão. A atividade específica das enzimas maltase e a sacarase aumenta rapidamente entre 19 e 21 dias de incubação, enquanto, após a eclosão, a atividade dessas enzimas continua a aumentar até o abate das aves (Chotinsky et al., 2001). Moran Jr. (1985) concluiu que as aves são capazes de digerir amido logo após a eclosão, ao passo que Uni et al. (1995) descreveram que, aos 4 dias de idade, as aves possuem completa capacidade para digestão do amido.

Neste estudo, objetivou-se a determinação da energia metabolizável aparente corrigida (EMAn) de alguns ingredientes como fonte de carboidrato para frangos de corte no período de 1 a 7 dias de idade, bem como avaliar a utilização destes diferentes ingredientes em dietas pré-iniciais, sobre o desenvolvimento inicial de órgãos do trato gastrintestinal e desempenho das aves de 1 a 7 e de 8 a 21 dias de idade. 


\section{Material e Métodos}

Dois experimentos foram realizados com frangos de corte nas fases pré-inicial e inicial em sala climatizada do Laboratório de Nutrição e Crescimento Animal do Departamento de Zootecnia da ESALQ/ USP de Piracicaba, sendo avaliadas quatro fontes de carboidratos: amido de milho ${ }^{1}$ (AMI), amido de mandioca $^{2}$ (AMA), glicose ${ }^{3}$ (GLI) e sacarose ${ }^{4}$ (SAC).

\section{Experimento 1}

Foi realizado um ensaio de metabolismo, utilizando-se 220 pintos de corte machos (AgRoss) de 1 a 7 dias de idade, distribuídos em delineamento inteiramente casualizado com cinco tratamentos e quatro repetições de 11 aves cada, alojadas em baterias com aquecimento automático. Sob o piso de arame havia bandejas revestidas com plástico, adequadas para coleta de excretas.

Os tratamentos consistiram de uma dieta-referência à base de milho e farelo de soja e de cinco dietas com $80 \%$ de dieta-referência e $20 \%$ dos ingredientes (AMI, AMA, GLI e SAC) como fonte de carboidrato. A dieta-referência foi formulada de acordo com os níveis nutricionais propostos por Rostagno et al. (2000) (Tabela 1).

O período de 1 a 3 dias de vida das aves foi considerado como fase de adaptação às instalações e dietas experimentais. No intervalo de 4 a 7 dias de idade, foi utilizado o método de coleta total de excretas, em que as quantidades da dieta ingerida e excreta produzida durante os quatro dias foram registradas.

As excretas foram coletadas duas vezes ao dia, identificadas e congeladas diariamente, sendo posteriormente descongeladas e homogeneizadas. Depois da retirada de amostras representativas para cada repetição, elas foram secas em estufa com circulação de ar a $55^{\circ} \mathrm{C}$ por 72 horas e preparadas para análises posteriores segundo metodologia proposta por Silva (1990). As amostras dos ingredientes, dietas experimentais e excretas foram submetidas à determinação da matéria seca, nitrogênio, extrato etéreo e energia bruta.

Os resultados obtidos nas análises para a dieta ingerida e excreta produzida foram utilizados para

\footnotetext{
${ }^{1}$ AMISOL ${ }^{\circledR} 3408$ (Corn Products Brasil).

2 Fécula de mandioca comercial.

3 Xarope de glicose MOR-REX® 1940 (Corn Products Brasil).

${ }^{4}$ Açúcar de cana cristal.
}

\section{R. Bras. Zootec., v.34, n.1, p.123-133, 2005}

Tabela 1- Composição percentual e valores nutricionais das dietas experimentais nas fases pré-inicial (Experimento 1) e inicial (Experimento 2)

Table 1- Percentage composition and nutritional values of experimental diets on newly hatched (Trial 1) and starter phases (Trial 2)

\begin{tabular}{|c|c|c|}
\hline $\begin{array}{l}\text { Ingredientes } \\
\text { Ingredients }\end{array}$ & $\begin{array}{c}\text { Pré-inicial } \\
\text { (experimento 1) } \\
\text { Newly hatched } \\
\text { (trial 1) }\end{array}$ & $\begin{array}{cc} & \text { Inicial } \\
& \text { (experimento 2) } \\
& \text { Starter } \\
& (\text { trial 2) } \\
(\%) & \end{array}$ \\
\hline Milho & 57,30 & 62,60 \\
\hline $\begin{array}{l}\text { Corn } \\
\text { Farelo de soja } \\
\text { Soybean meal }\end{array}$ & 36,75 & 32,50 \\
\hline $\begin{array}{l}\text { Fosfato bicálcico } \\
\text { Dicalcium phosphate }\end{array}$ & 1,89 & 1,76 \\
\hline $\begin{array}{l}\text { Calcário calcítico } \\
\text { Limestone }\end{array}$ & 1,00 & 1,12 \\
\hline $\begin{array}{l}\text { Sal } \\
\text { Salt }\end{array}$ & 0,50 & 0,40 \\
\hline $\begin{array}{l}\text { DL-Metionina } \\
\text { DL-methionine }\end{array}$ & 0,21 & 0,17 \\
\hline $\begin{array}{l}\text { L-lisina. } \mathrm{HCl} \\
\text { L-lysine }\end{array}$ & 0,18 & 0,20 \\
\hline $\begin{array}{l}\text { Cloreto de colina }-60 \% \\
\text { Choline chloride }\end{array}$ & 0,05 & 0,05 \\
\hline $\begin{array}{l}\text { Óleo vegetal } \\
\text { Vegetable oil }\end{array}$ & 1,92 & 1,12 \\
\hline $\begin{array}{l}\text { Suplemento vitamínico } \\
\text { Vitamin mix }\end{array}$ & 0,10 & 0,08 \\
\hline $\begin{array}{l}\text { Suplemento mineral } \\
\text { Mineral mix }^{2}\end{array}$ & 0,10 & 0,10 \\
\hline $\begin{array}{l}\text { Composição calculada } \\
\text { Calculated composition }\end{array}$ & & \\
\hline $\begin{array}{l}\mathrm{EM}(\mathrm{kcal} / \mathrm{kg}) \\
M E\end{array}$ & 2.950 & 3.050 \\
\hline $\begin{array}{l}\mathrm{PB}(\%) \\
C P\end{array}$ & 21,92 & 21,22 \\
\hline $\begin{array}{l}\text { Metionina }(\%) \\
\text { Methionine }\end{array}$ & 0,54 & 0,49 \\
\hline $\begin{array}{l}\text { Metionina }+ \text { cistina }(\%) \\
\text { Methionine }+ \text { cystine }\end{array}$ & 0,97 & 0,85 \\
\hline $\begin{array}{l}\text { Lisina }(\%) \\
\text { Lysine }\end{array}$ & 1,31 & 1,26 \\
\hline $\begin{array}{l}\text { Arginina }(\%) \\
\text { Arginine }\end{array}$ & 1,45 & 1,36 \\
\hline $\begin{array}{l}\text { Treonina }(\%) \\
\text { Threonine }\end{array}$ & 0,84 & 0,82 \\
\hline $\begin{array}{l}\text { Triptofano }(\%) \\
\text { Tryptophan }\end{array}$ & 0,27 & 0,25 \\
\hline $\begin{array}{l}\text { Fósforo disponível (\%) } \\
\text { Available phosphorus }\end{array}$ & 0,47 & 0,45 \\
\hline $\begin{array}{l}\text { Cálcio }(\%) \\
\text { Calcium }\end{array}$ & 0,99 & 0,96 \\
\hline
\end{tabular}

${ }^{1}$ Quantidade por kg de ração nos experimentos 1 e 2, respectivamente (supplying per $\mathrm{kg}$ of feed in trials 1 and 2, respectively): vit. A, 10.000 e $8.000 \mathrm{UI}$; vit. $\mathrm{D}_{3}, 2.000$ e $1.600 \mathrm{UI}$; vit. E, $12,5 \mathrm{e}$ $10 \mathrm{mg}$; vit. $\mathrm{K}_{3}$, 2,5 e $2 \mathrm{mg}$; vit. $\mathrm{B}_{1}, 2,4$ e 1,92 mg; vit. $\mathrm{B}_{2}, 6,0$ e $4,8 \mathrm{mg}$; vit. $B_{6}, 3,2$ e 2,56 mg; vit. $B_{12}, 12$ e 9,6 $\mu$ g; ácido fólico (folic acid), 1,0 e 0,8 mg; pantotenato de $\mathrm{Ca}$ (calcium pantotenate), 12,5 e $10 \mathrm{mg}$; niacina (niacin), 30 e $24 \mathrm{mg}$; selênio (selenium), 0,2 e $0,16 \mathrm{mg}$; BHT, 15 e $12 \mathrm{mg}$.

${ }^{2}$ Quantidade por kg de ração (supplying per $\mathrm{kg}$ of feed): manganês (manganese), $65 \mathrm{mg}$; cobre (copper), $12 \mathrm{mg}$; zinco (zinc), $50 \mathrm{mg}$; ferro (iron), $40 \mathrm{mg}$; iodo (iodine), $1 \mathrm{mg}$. 
determinar a energia metabolizável aparente corrigida para o balanço de nitrogênio (EMAn) das dietas experimentais, de acordo com Matterson et al. (1965). A partir dos valores de EMAn determinados para as dietas experimentais, foi possível calcular os valores de EMAn de cada ingrediente-teste:

$$
\text { EMAn ing. }=\frac{\text { EMAn ref. }+(\text { EMAn teste }- \text { EMAn ref. })}{(\% \text { substituição } / 100)}
$$

em queEMAn - energia metabolizável aparente corrigida pelo balanço de nitrogênio calculada ( $\mathrm{kcal} / \mathrm{kg})$; ing. ingrediente testado; ref. - dieta-referência; teste - dieta basal + ingrediente-teste; \% substituição - nível de substituição da dieta basal pelo ingrediente-teste.

A partir dos valores de energia bruta (EB) e da EMAn dos ingredientes, foram calculados os coeficientes de metabolizabilidade da energia bruta $(\mathrm{CMEB}=(\mathrm{EMAn} / \mathrm{EB}) \times 100)$.

\section{Experimento 2}

Foram utilizados 520 pintos de corte machos de um dia de idade, marca comercial AgRoss, provenientes de matrizes com 62 semanas de idade, alojados em baterias com aquecimento automático, distribuídos em delineamento inteiramente casualizado com cinco tratamentos e quatro repetições de 26 aves.

Os tratamentos foram aplicados de 1 a 7 dias, sendo que no período subsequiente ( 8 a 21 dias), as aves tiveram acesso a uma dieta basal (Tabela 1). Os tratamentos consistiram em: 1 - dieta de milho e farelo de soja (testemunha), 2 - dieta de milho e farelo de soja + AMI, 3 - dieta de milho e farelo de soja + AMA, 4 - dieta de milho e farelo de soja + GLI, 5 - dieta de milho e farelo de soja + SAC.

As dietas experimentais fornecidas nos experimentos para avaliação dos ingredientes, como fonte de carboidratos na fase pré-inicial (Tabela 2), foram formuladas para atender às exigências nutricionais propostas por Rostagno et al. (2000), sendo apenas ajustados os valores de EM para esta fase, de 2.950 para $2.800 \mathrm{kcal} / \mathrm{kg}$. Esse ajuste foi considerado aplicando-se os valores de EMAn determinados para o milho e farelo de soja na fase pré-inicial (Menten et al., 2002).

Para a utilização das fontes de carboidrato, foi considerado o valor de energia metabolizável determinado no Experimento 1 de modo que o mesmo participou na dieta contribuindo com $20 \%$ do valor de EM total.

Nos períodos de 1 a 7 e de 8 a 21 dias, foram registrados o consumo de ração, ganho de peso, conversão alimentar e viabilidade criatória. Aos 1,7 e 21 dias de idade, as aves foram pesadas individualmente para o cálculo de uniformidade (coeficiente de variação do peso individual das aves da parcela) da parcela.

Antes do alojamento, quatro aves foram retiradas para avaliação inicial dos órgãos (dia zero). Aos 1, 4 e 7 dias de idade, foram retiradas aleatoriamente duas aves por parcela, que foram abatidas por deslocamento cervical e os órgãos retirados para mensurações. Foram retirados e determinados o peso relativo (\% do peso vivo) do saco vitelino residual, proventrículo, moela, pâncreas, fígado e o peso relativo, comprimento $(\mathrm{cm})$ e densidade $(\mathrm{mg} / \mathrm{cm})$ do intestino delgado vazio.

Os coeficientes de metabolizabilidade da energia bruta dos ingredientes avaliados, os resultados de desempenho e desenvolvimento dos órgãos do trato gastrintestinal foram submetidos à análise de variância pelo PROC GLM do programa Statistical Analysis System (SAS, 1996). As médias obtidas foram comparadas pelo teste Tukey e as diferenças, consideradas significativas quando $\mathrm{P}<0,05$ para o coeficiente de metabolizabilidade e desempenho das aves e $\mathrm{P}<0,10$ para os dados de desenvolvimento dos órgãos do trato gastrintestinal. 
Tabela 2 - Composição percentual e valores nutricionais das dietas experimentais na fase pré-inicial para avaliação das fontes de carboidratos (Experimento 2)

Table 2 - Percentage composition and nutritional values of experimental diets to evaluate carbohydrate sources for newly hatched chicks (Trial 2)

\begin{tabular}{|c|c|c|c|c|c|}
\hline $\begin{array}{l}\text { Ingredientes } \\
\text { Ingredients }\end{array}$ & $\begin{array}{c}\text { Testemunha } \\
\text { (TES) } \\
\text { Control }\end{array}$ & $\begin{array}{l}\text { Glicose } \\
\text { (GLI) } \\
\text { Glucose }\end{array}$ & $\begin{array}{l}\text { Sacarose } \\
\text { (SAC) } \\
\text { Sucrose }\end{array}$ & $\begin{array}{c}\text { Amido de milho } \\
\text { (AMI) } \\
\text { Corn starch }\end{array}$ & $\begin{array}{c}\text { Amido de mandioca } \\
\text { (AMA) } \\
\text { Cassava starch }\end{array}$ \\
\hline $\begin{array}{l}\text { Milho }^{1} \\
\text { Corn }\end{array}$ & 61,19 & 41,03 & 41,79 & 39,58 & 43,27 \\
\hline $\begin{array}{l}\text { Farelo de soja }{ }^{1} \\
\text { Soybean meal }\end{array}$ & 33,57 & 37,35 & 37,27 & 37,48 & 36,97 \\
\hline $\begin{array}{l}\text { Carboidrato teste } \\
\text { Test carbohydrate }\end{array}$ & - & 16,34 & 15,89 & 17,13 & 15,18 \\
\hline $\begin{array}{l}\text { Fosfato bicálcico } \\
\text { Dicalcium phosphate }\end{array}$ & 1,86 & 1,90 & 1,90 & 1,90 & 1,89 \\
\hline $\begin{array}{l}\text { Calcário calcítico } \\
\text { Limestone }\end{array}$ & 1,04 & 0,97 & 1,00 & 0,99 & 1,00 \\
\hline $\begin{array}{l}\text { Óleo vegetal } \\
\text { Vegetable oil }\end{array}$ & 1,29 & 1,43 & 1,17 & 1,93 & 0,70 \\
\hline $\begin{array}{l}\text { Sal } \\
\text { Salt }\end{array}$ & 0,40 & 0,40 & 0,40 & 0,40 & 0,40 \\
\hline $\begin{array}{l}\text { DL-Metionina } \\
\text { DL-methionine }\end{array}$ & 0,18 & 0,19 & 0,19 & 0,19 & 0,19 \\
\hline $\begin{array}{l}\mathrm{L} \text {-lisina. } \mathrm{HCl}(\text { L-lysine }) \\
\text { Cloreto de colina } 60 \%\end{array}$ & $\begin{array}{l}0,22 \\
0,05\end{array}$ & $\begin{array}{l}0,14 \\
0,05\end{array}$ & $\begin{array}{l}0,14 \\
0,05\end{array}$ & $\begin{array}{l}0,14 \\
0,05\end{array}$ & $\begin{array}{l}0,15 \\
0,05\end{array}$ \\
\hline $\begin{array}{l}\text { Choline chloride } \\
\text { Supl. vitamínico } \\
\text { Vitamin mix } \\
\end{array}$ & 0,10 & 0,10 & 0,10 & 0,10 & 0,10 \\
\hline $\begin{array}{l}\text { Supl. mineral } \\
\text { Mineral mix }\end{array}$ & 0,10 & 0,10 & 0,10 & 0,10 & 0,10 \\
\hline $\begin{array}{l}\text { Composição calculada } \\
\text { Calculated composition }\end{array}$ & & & & & \\
\hline $\begin{array}{l}\mathrm{EM}(\mathrm{kcal} / \mathrm{kg})(M E) \\
\mathrm{PB}(\%)(C P) \\
\text { Metionina }(\%) \\
\text { Methionine }\end{array}$ & $\begin{array}{c}2.800 \\
21,92 \\
0,51\end{array}$ & $\begin{array}{c}2.800 \\
21,92 \\
0,51\end{array}$ & $\begin{array}{c}2.800 \\
21,92 \\
0,51\end{array}$ & $\begin{array}{c}2.800 \\
21,92 \\
0,51\end{array}$ & $\begin{array}{c}2.800 \\
21,92 \\
0,51\end{array}$ \\
\hline $\begin{array}{l}\text { Metionina+cistina (\%) } \\
\text { Methionine+cystine }\end{array}$ & 0,87 & 0,86 & 0,86 & 0,86 & 0,86 \\
\hline $\begin{array}{l}\text { Lisina }(\%) \\
\text { Lysine }\end{array}$ & 1,31 & 1,31 & 1,31 & 1,31 & 1,31 \\
\hline $\begin{array}{l}\text { Arginina (\%) } \\
\text { Arginine }\end{array}$ & 1,39 & 1,44 & 1,44 & 1,44 & 1,44 \\
\hline $\begin{array}{l}\text { Treonina }(\%) \\
\text { Threonine }\end{array}$ & 0,83 & 0,84 & 0,84 & 0,84 & 0,84 \\
\hline $\begin{array}{l}\text { Triptofano }(\%) \\
\text { Tryptophan }\end{array}$ & 0,26 & 0,27 & 0,27 & 0,27 & 0,27 \\
\hline $\begin{array}{l}\text { Fósforo disponível (\%) } \\
\text { Available phosphorus }\end{array}$ & 0,47 & 0,47 & 0,47 & 0,47 & 0,47 \\
\hline $\begin{array}{l}\text { Cálcio }(\%) \\
\text { Calcium }\end{array}$ & 0,99 & 0,99 & 0,99 & 0,99 & 0,99 \\
\hline
\end{tabular}


Tabela 3 - Valores determinados de matéria seca (MS), energia bruta (EB), proteína bruta (PB) e extrato etéreo (EE) dos ingredientes

Table 3 - Determined values for dry matter (MS), gross energy $(E B)$, crude protein $(C P)$ and ether extract $(E E)$ of the ingredients

\begin{tabular}{lcccc}
\hline $\begin{array}{l}\text { Ingredientes } \\
\text { Ingredients }\end{array}$ & $\begin{array}{c}\mathrm{MS} \\
(\%)\end{array}$ & $\begin{array}{c}\mathrm{EB} \\
(\mathrm{kcal} / \mathrm{kg}) *\end{array}$ & $\begin{array}{c}\mathrm{PB} \\
(\%)^{*}\end{array}$ & $\begin{array}{c}\mathrm{EE} \\
(\%)^{*}\end{array}$ \\
\hline $\begin{array}{l}\text { Amido de milho } \\
\begin{array}{l}\text { Corn starch } \\
\text { Amido de mandioca }\end{array}\end{array}$ & 89,30 & 3.619 & 1,43 & 0,36 \\
$\begin{array}{l}\text { Cassava starch } \\
\begin{array}{l}\text { Glicose } \\
\text { Glucose }\end{array}\end{array}$ & 97,81 & 3.770 & 0,87 & 1,72 \\
$\begin{array}{l}\text { Sacarose } \\
\text { Sucrose }\end{array}$ & 93,17 & 3.819 & 1,03 & 0,86 \\
\hline
\end{tabular}

*Valores expressos na matéria natural (Values as fed basis).

\section{Resultados e Discussão}

\section{Experimento 1}

Os valores analisados de matéria seca (MS), energia bruta (EB), proteína bruta $(\mathrm{PB})$ e extrato etéreo (EE) dos ingredientes avaliados (Tabela 3) são coerentes com os valores encontrados em tabelas de composição.

Na Tabela 4, são apresentados os valores calculados de EMAn e CMEB dos ingredientes avaliados no ensaio de metabolismo, além de valores de EMA descritos na literatura. Verifica-se que os valores de EMAn para pintos de 1 a 7 dias de idade foram superiores para a glicose, semelhantes para o amido de milho e inferiores para a sacarose, quando comparados aos apresentados na literatura (NRC, 1994; Rostagno et al., 2000).

Essa maior EMAn encontrada para glicose, na fase pré-inicial, pode estar relacionada ao maior aproveitamento deste monossacarídeo, corroborando os relatos de Batal \& Parsons (2003). Segundo esses autores, a obtenção de alto valor de EMAn para uma dieta composta por dextrose-caseína para frangos jovens decorreu do alto valor de contribuição energética da glicose, indicando que esse ingrediente é muito bem aproveitado por aves neonatas.

O elevado valor de EMAn para o amido de mandioca e o valor semelhante do amido de milho para aves durante a primeira semana de vida, em comparação aos valores descritos na literatura (aves em idades mais avançadas), demonstra a capacidade precoce de digestão do amido em aves. Esses resultados comprovam as evidências apresentadas na
Tabela 4 - Valores calculados e seus respectivos desvios-padrão para a energia metabolizável aparente corrigida pelo balanço de nitrogênio (EMAn) e o coeficiente de metabolizabilidade da energia bruta (CMEB) dos ingredientes avaliados no ensaio experimental

Table 4 - Calculated values and respective standard deviations for $\mathrm{N}$-corrected apparent metabolizable energy (EMAn) and metabolizability coefficient of gross energy (CMEB) of the ingredients evaluated in the experimental trial

\begin{tabular}{lccc}
\hline $\begin{array}{l}\text { Ingredientes } \\
\text { Ingredients }\end{array}$ & $\begin{array}{c}\mathrm{EMAn}^{1} \\
(\mathrm{kcal} / \mathrm{kg} \mathrm{MN})\end{array}$ & $\begin{array}{c}\text { CMEB } \\
(\%)\end{array}$ & $\begin{array}{r}\text { EMAn literatura } \\
(\mathrm{kcal} / \mathrm{kg} \mathrm{MN})\end{array}$ \\
\hline $\begin{array}{l}\text { Amido de } \\
\text { milho }\end{array}$ & $3.269 \pm 0,105$ & $90,33 \pm 2,89 \mathrm{ab}$ & $3.201-3.625$ \\
$\begin{array}{l}\text { Corn starch } \\
\text { Amido de }\end{array}$ & & & \\
mandioca & $3.690 \pm 0,154$ & $97,89 \pm 4,07 \mathrm{a}$ & - \\
$\begin{array}{l}\text { Cassava starch } \\
\text { Glicose }\end{array}$ & $3.427 \pm 0,099$ & $89,74 \pm 2,58 \mathrm{~b}$ & $3.168-3.326$ \\
$\begin{array}{l}\text { Glucose } \\
\text { Sacarose }\end{array}$ & $3.524 \pm 0,206$ & $89,85 \pm 5,24 \mathrm{ab}$ & 3.831 \\
Sucrose & & & \\
\hline
\end{tabular}

${ }^{1} \mathrm{MN}$ = na matéria natural (as fed basis).

${ }^{2}$ NRC (1994), Rostagno et al. (2000).

a, b. Médias seguidas de letras diferentes na mesma coluna diferem entre si pelo teste Tukey $(\mathrm{P}<0,05)$ (Average values followed by different letters in the same column are different by Tukey test $[P<.05])$.

Coeficiente de variação $=4,18 \%$ (coefficient of variation $=4.18 \%$ ).

literatura em relação à capacidade de digerir o carboidrato amido logo após a eclosão e sobretudo após 4 dias de idade (Uni et al., 1995; Moran Jr., 1985).

Para a sacarose verificou-se um valor de EMAn cerca de $300 \mathrm{kcal} / \mathrm{kg}$ inferior ao valor proposto por Rostagno et al. (2000). Essa diferença pode estar associada à menor atividade da enzima sacarase ainda na fase jovem, uma vez que existem evidências de que, se o carboidrato sacarose fosse hidrolisado pela enzima, a glicose derivada teria maior taxa de absorção em relação à forma livre fornecida e, conseqüentemente, melhor aproveitamento energético (Levin, 1976).

Apesar do alto valor energético da glicose, verifica-se que o coeficiente de metabolizabilidade foi significativamente menor $(\mathrm{P}<0,05)$ em relação ao amido de mandioca, sendo que o amido de milho e a sacarose apresentaram valores intermediários.

Alto valor de metabolizabilidade foi observado para o amido de mandioca até sete dias de idade, ressaltando a importância de se verificar os efeitos da utilização deste ingrediente sobre o desempenho e desenvolvimento dos órgãos em aves neonatas. 
A partir dos valores encontrados nas tabelas brasileiras de composição de alimentos (Rostagno et al., 2000), pode-se calcular o coeficiente de metabolizabilidade de alguns dos ingredientes avaliados no presente experimento e verificar que o aproveitamento energético da glicose e sacarose na fase pré-inicial (Tabela 4) é menor que em aves adultas $(98,4$ e $95,7 \%$, respectivamente). Por outro lado, para o amido de milho (Tabela 4), os valores são mais próximos aos da literatura $(93,6 \%)$. Estes resultados concordam com Mateos \& Sell (1980), os quais observaram que a adição de açúcares simples na dieta pré-inicial promove taxa de passagem mais rápida por todo o TGI em relação a dietas com amido. Conseqüentemente, a digestibilidade de açúcares simples é menor que a do amido.

A diferença encontrada entre os valores determinados neste ensaio e os da literatura indica que as características metabólicas existentes em cada fase de desenvolvimento podem afetar o valor energético dos alimentos e, conseqüentemente, alterar o valor de energia metabolizável fornecido na dieta.

\section{Experimento 2}

Os valores médios dos tratamentos para as variáveis de desempenho (ganho de peso, consumo de ração e conversão alimentar), uniformidade e viabilidade criatória na fase de 1 a 7 dias de idade são apresentados na Tabela 5. Observou-se efeito significativo $(\mathrm{P}<0,05)$ dos tratamentos somente sobre o consumo de ração das aves, sendo que as aves que receberam o carboidrato GLI tiveram maior consumo em relação às aves dos tratamentos AMI e TES. O consumo de ração em aves durante a fase pré-inicial pode estar relacionada à capacidade de digestão da dieta de modo que esse consumo não exceda a capacidade digestiva (Noy \& Sklan, 199).

De maneira geral, foi observado maior ganho de peso das aves alimentadas nessa fase com as diferentes fontes de carboidrato (AMA, AMI, GLI e SAC), em relação à testemunha, sendo que a maior diferença, apesar de também não-significativa $(\mathrm{P}>0,05)$, foi cerca de $14 \mathrm{~g} / \mathrm{ave}$, ou $11 \%$, entre o tratamento GLI e TES. Este resultado pode ser explicado pelas diferenças no consumo de ração das aves, que foi significativo $(\mathrm{P}<0,05)$ para GLI. Apesar da diferença nãosignificativa $(\mathrm{P}>0,05)$, os tratamentos AMI, AMA e SAC promoveram maior consumo de ração das aves em relação ao tratamento TES. A conversão alimentar das aves nesse período não diferiu entre os tratamentos $(\mathrm{P}>0,05)$.

Não houve efeito significativo $(\mathrm{P}>0,05)$ dos tratamentos sobre a uniformidade e viabilidade criatória de 1 a 7 dias de idade das aves.

Na Tabela 6 são apresentados os valores médios de peso relativo do saco vitelino, proventrículo e moela aos 1, 4 e 7 dias de idade dos frangos dos diferentes tratamentos. A absorção do saco vitelino das aves que receberam as diferentes fontes de carboidratos não diferiu significativamente $(\mathrm{P}>0,10)$, o que deve estar relacionado com o elevado valor do coeficiente de variação.

Tabela 5 - Valores médios de ganho de peso (GP), consumo de ração (CR), conversão alimentar (CA), uniformidade (UNIF) e viabilidade criatória (VIAB) de frangos de corte de 1 a 7 dias de idade alimentados com diferentes fontes de carboidrato

Table 5 - Average values of weight gain (GP), feed intake (CR), feed conversion (CA), uniformity (UNIF) and viability (VIAB) of the broilers from 1 to 7 days fed different carbohydrate sources

\begin{tabular}{|c|c|c|c|c|c|c|}
\hline & $\begin{array}{c}\mathrm{GP} \\
\text { (g/ave) } \\
(\text { g/bird) }\end{array}$ & $\begin{array}{c}\mathrm{CR} \\
\text { (g/ave) } \\
\text { (g/bird) } \\
\end{array}$ & $\begin{array}{l}\mathrm{CA} \\
(\mathrm{g} / \mathrm{g}) \\
\end{array}$ & $\begin{array}{c}\mathrm{UNIF}^{1} \\
\text { Inicial(initial) } \\
(\%) \\
\end{array}$ & $\begin{array}{c}\mathrm{UNIF}^{1} \\
7 \text { dias (7 days) } \\
(\%) \\
\end{array}$ & $\begin{array}{c}\text { VIAB } \\
1 \text { a } 7 \text { dias }(1 \text { to } 7 \text { days }) \\
(\%) \\
\end{array}$ \\
\hline TES & 127,3 & $141,1 \mathrm{~b}$ & 1,11 & 8,7 & 12,0 & 96,2 \\
\hline AMI & 132,6 & $142,1 \mathrm{~b}$ & 1,07 & 9,0 & 10,9 & 97,1 \\
\hline AMA & 136,0 & $147,2 \mathrm{ab}$ & 1,08 & 8,1 & 10,2 & 97,1 \\
\hline GLI & 141,8 & $157,5 \mathrm{a}$ & 1,11 & 9,0 & 8,0 & 97,1 \\
\hline SAC & 134,7 & $149,4 \mathrm{ab}$ & 1,11 & 9,7 & 10,8 & 96,2 \\
\hline $\mathrm{CV}(\%)$ & 5,27 & 4,07 & 3,07 & 15,97 & 27,03 & 2,12 \\
\hline
\end{tabular}

a, b. Médias seguidas de diferentes letras na coluna diferem pelo teste Tukey $(\mathrm{P}<0,05)$ (Average values followed by different letters in the same column differ each other by Tuket test $[P<.05])$.

1 Média do coeficiente de variação dos pesos individuais das aves de cada parcela nos diferentes tratamentos (Average value of coefficient of variation of individual live weight of birds of each pen in the different treatments).

$\mathrm{CV}(\%)=$ coeficiente de variação (coefficient of variation).

R. Bras. Zootec., v.34, n.1, p.123-133, 2005 
Tabela 6 - Peso relativo (\% do peso vivo) do saco vitelino, proventrículo e moela aos 1, 4 e 7 dias de idade para frangos alimentados com diferentes fontes de carboidrato na dieta pré-inicial

Table 6 - Relative weight (\% of live weight) of yolk sac, proventriculum and gizzard at 1, 4 and 7 days of age for broilers fed different carbohydrate sources in the diets

\begin{tabular}{|c|c|c|c|c|c|c|c|c|c|}
\hline & \multicolumn{3}{|c|}{$\begin{array}{c}\text { Saco vitelino } \\
\text { Yolk sac }\end{array}$} & \multicolumn{3}{|c|}{$\begin{array}{l}\text { Proventrículo } \\
\text { Proventriculum }\end{array}$} & \multicolumn{3}{|c|}{$\begin{array}{l}\text { Moela } \\
\text { Gizzard }\end{array}$} \\
\hline & $\begin{array}{l}1 \mathrm{dia} \\
1 \text { day }\end{array}$ & $\begin{array}{l}4 \text { dias } \\
4 \text { days }\end{array}$ & $\begin{array}{l}7 \text { dias } \\
7 \text { days }\end{array}$ & $\begin{array}{l}1 \text { dia } \\
1 \text { day }\end{array}$ & $\begin{array}{l}4 \text { dias } \\
4 \text { days }\end{array}$ & $\begin{array}{l}7 \text { dias } \\
7 \text { days }\end{array}$ & $\begin{array}{l}1 \mathrm{dia} \\
1 \mathrm{day}\end{array}$ & $\begin{array}{l}4 \text { dias } \\
4 \text { days }\end{array}$ & $\begin{array}{l}7 \text { dias } \\
7 \text { days }\end{array}$ \\
\hline TES & 5,73 & 0,90 & 0,21 & 1,34 & 1,53 & 1,05 & 5,24 & 5,31 & 4,32 \\
\hline AMI & 5,88 & 0,49 & 0,16 & 1,05 & 1,50 & 1,06 & 5,40 & 5,14 & 4,21 \\
\hline AMA & 5,63 & 0,89 & 0,18 & 1,33 & 1,38 & 1,10 & 5,56 & 5,12 & 4,10 \\
\hline GLI & 6,31 & 0,52 & 0,15 & 1,10 & 1,34 & 1,27 & 5,44 & 4,85 & 4,12 \\
\hline SAC & 5,56 & 1,14 & 0,29 & 1,22 & 1,33 & 1,11 & 5,63 & 4,96 & 4,39 \\
\hline $\mathrm{CV}(\%)$ & 24,82 & 71,64 & 55,02 & 15,94 & 7,48 & 13,17 & 6,45 & 5,48 & 8,51 \\
\hline
\end{tabular}

Considerando-se que as aves, ao serem alojadas, apresentavam um saco vitelino de $10,23 \%$ do peso vivo $(5,34 \mathrm{~g})$, observa-se rápida absorção do conteúdo do saco já nas primeiras 24 horas (média de $5,82 \%$ do peso vivo ou $3,3 \mathrm{~g}$ ). Aos 4 dias de idade, o peso relativo do saco vitelino corresponde a menos de $1 \%$ do peso vivo da ave, exceto para as aves do tratamento SAC $(1,14 \%)$. Essa absorção dos nutrientes do saco vitelino no intestino delgado é fundamental para o crescimento inicial da ave e serve como estímulo ao desenvolvimento do TGI e, conseqüentemente, das funções de digestão e absorção (Iji et al., 2001a).

O desenvolvimento do proventrículo das aves não sofreu influência $(\mathrm{P}>0,10)$ dos diferentes carboidratos fornecidos. A função digestiva do proventrículo está mais relacionada à proteína, sendo que ocorre nesse órgão apenas por uma pequena hidrólise química dos carboidratos a partir do $\mathrm{HCl}$ produzido. De maneira geral, o proventrículo das aves, no momento do alojamento, pesava $0,43 \mathrm{~g}(0,82 \%$ do peso vivo), desenvolvendo-se mais em relação ao peso vivo das aves até os 4 dias de idade $(1,41 \%)$.

Esperar-se-ia, para moela (estômago mecânico das aves), pequena variação em função do tamanho de partículas das dietas, uma vez que maiores quantidades de partículas finas, como AMI, AMA e GLI, exigiriam menor atividade de maceração e, conseqüentemente, menor peso relativo da moela. Entretanto, não foi observado efeito de tratamento $(\mathrm{P}>0,10)$ sobre o peso relativo da moela aos 1,4 e 7 dias de idade. As aves apresentaram, no dia zero, peso de moela de $4,93 \%$ do peso vivo $(2,57 \mathrm{~g})$. Considerando peso médio entre os diferentes tratamentos, observou-se desenvolvimento maior deste órgão que o peso da ave nas primeiras 24 horas $(5,45 \%$ do peso vivo) e, após, continuou a se desenvolver, mas em proporções menores que o peso vivo da ave.

Para o desenvolvimento do pâncreas das aves (Tabela 7), não foi observado efeito das diferentes fontes de carboidratos $(\mathrm{P}>0,10)$. $\mathrm{O}$ peso do pâncreas pode representar mudanças na capacidade digestiva da ave, em virtude da alta correlação entre o peso do mesmo com a atividade das enzimas digestivas pancreáticas (Corless \& Sell, 1999). Em função disso, esperava-se redução no tamanho relativo desse órgão das aves alimentadas com GLI, devido à presença de glicose disponível, sem necessidade de enzimas para o processo de digestão, além de aumento do peso relativo do órgão das aves que receberam os tratamentos AMA e AMI, em função da maior concentração do carboidrato amido. De maneira geral, o pâncreas apresentou um peso de $0,17 \%$ do peso vivo $(0,09 \mathrm{~g})$ no dia de alojamento, aumentando em três vezes mais em relação ao peso da ave até os 4 dias de idade $(0,51 \%)$, após esse período, o crescimento do pâncreas acompanhou o crescimento da ave.

O pâncreas desenvolve-se rapidamente após a eclosão, enfatizando a importância deste órgão para as aves neonatas (Katanbaf et al., 1988). Nitsan et al. (1995) relatou que, durante a primeira semana de vida dos pintos de corte, o pâncreas aumenta quatro vezes e o fígado duas vezes mais que o peso do corpo.

O fígado das aves (Tabela 7) aumentou de 2,78\% $(1,45 \mathrm{~g})$ para $5,40 \%$ do peso vivo no período entre o alojamento e 4 dias de idade. Após 24 horas, não se observou efeito significativo $(\mathrm{P}>0,10)$ dos carboidratos sobre o peso relativo do fígado. Aos 4 dias de idade, verificou-se que o tratamento com AMA proporcionou maior $(\mathrm{P}<0,10)$ peso relativo desse órgão das aves em relação ao tratamento AMI. 
Tabela 7 - Peso relativo (\% do peso vivo) do pâncreas e fígado aos 1, 4 e 7 dias de idade para frangos alimentados com diferentes fontes de carboidrato na dieta pré-inicial

Table 7 - Relative weight (\% of live weight) of pancreas and liver at 1, 4 and 7 days of age for broilers fed different carbohydrate sources in the diets

\begin{tabular}{|c|c|c|c|c|c|c|}
\hline & \multicolumn{3}{|c|}{ Pâncreas (pancreas) } & \multicolumn{3}{|c|}{ Fígado (liver) } \\
\hline & 1 dia & 4 dias & 7 dias & $1 \mathrm{dia}$ & 4 dias & 7 dias \\
\hline & 1 day & 4 days & 7 days & 1 day & 4 days & 7 days \\
\hline TES & 0,32 & 0,47 & 0,48 & 3,88 & $5,62 \mathrm{ab}$ & 4,57 \\
\hline AMI & 0,27 & 0,51 & 0,50 & 3,49 & $4,83 b$ & 4,60 \\
\hline AMA & 0,29 & 0,55 & 0,53 & 3,57 & $5,74 a$ & 4,74 \\
\hline GL & 0,27 & 0,55 & 0,47 & 3,74 & $5,50 \mathrm{ab}$ & 5,17 \\
\hline SAC & 0,23 & 0,50 & 0,50 & 3,71 & $5,32 \mathrm{ab}$ & 5,17 \\
\hline $\mathrm{CV}(\%)$ & 17,34 & 20,15 & 11,35 & 7,89 & 8,55 & 6,62 \\
\hline
\end{tabular}

a, b. Médias seguidas de diferentes letras na coluna diferem entre si pelo teste Tukey $(\mathrm{P}<0,10)$ (Average values followed by different letters in the same column differ $[P<.10]$ by Tukey test).

Aos 7 dias, apesar das diferenças não-significativas $(\mathrm{P}>0,10)$, verificou-se tendência de maior peso relativo do fígado das aves que foram alimentadas com alguma fonte de carboidrato. As aves que receberam os tratamentos com os carboidratos GLI e SAC apresentaram valor $13 \%$ maior que as aves do tratamento somente com milho (TES). Essa diferença é superior ao aumento de $6 \%$, proposto por Guyton (1992), em relação à quantidade de hipertrofia hepática, em função da deposição de glicogênio.

Os resultados médios do desenvolvimento do intestino delgado aos 1 , 4 e 7 dias de idade são apresentados na Tabela 8. Houve efeito de tratamento $(\mathrm{P}<0,10)$ sobre o peso relativo do intestino delgado (ID) somente aos quatro dias de idade, sendo que as aves do tratamento AMI apresentaram maior peso do ID em relação às aves do tratamento GLI.

Considerando-se valor médio do peso relativo do ID dos tratamentos que receberam algum tipo de carboidrato (AMI, AMA, GLI e SAC), observou-se sempre maior peso relativo do ID das aves submetidas a esses tratamentos em relação ao tratamento TES.

No momento do alojamento, as aves apresentaram ID com peso de $2,64 \%$ do peso vivo $(1,38 \mathrm{~g})$, sendo que esse órgão aumentou pouco menos de duas vezes em relação ao peso da ave até os 7 dias de idade $(4,64 \%)$. Em termos de peso bruto, esse aumento correspondeu a, aproximadamente, $600 \%$, concordando com os relatos de Noy et al. (2001), que observaram que, com o estímulo do fornecimento de alimento, o peso do intestino delgado pode aumentar em até cerca de $600 \%$ nos primeiros sete dias.

Em relação ao comprimento do ID das aves, não se verificou efeito significativo $(\mathrm{P}>0,10)$ dos tratamentos nas diferentes idades. Notou-se rápido crescimento em tamanho do ID das aves nessa fase de criação, partindo de cerca de $39 \mathrm{~cm}$, ao alojamento, até cerca de $97 \mathrm{~cm}$ aos 7 dias de idade, ou seja, aumento de mais de duas vezes o tamanho inicial. Essa medida do comprimento do ID aos 7 dias corresponde a cerca de 2/3 do comprimento final aos 42 dias desse órgão (Sorbara, 2003), ressaltando a importância desse desenvolvimento inicial.

O comprimento do intestino delgado aumenta durante a primeira semana mesmo quando a ave não recebe alimento, mas, para o desenvolvimento das vilosidades, a alimentação é essencial (Baranyiovä \& Holman, 1976). Entretanto, também não foi observado efeito significativo $(\mathrm{P}>0,10)$ dos tratamentos sobre a densidade, ou seja, a relação peso:comprimento do ID. A densidade aumentou muito do primeiro (média de $36 \mathrm{mg} / \mathrm{cm}$ ) ao sétimo dia de vida da ave (média de $87 \mathrm{mg} / \mathrm{cm}$ ), resultado indireto do crescimento da mucosa intestinal, em que maiores densidades do intestino podem representar desenvolvimento em altura e diâmetro das vilosidades (Corless \& Sell, 1999; Uni et al., 1998) e, conseqüentemente, aumento da capacidade digestiva e absortiva.

Além do desenvolvimento das vilosidades, vários fatores podem estar contribuindo para o aumento da densidade do ID com o passar da idade: a quantidade de muco depositada, que pode ser influenciada pela composição da dieta; o estímulo para o desenvolvimento, influenciado pelo tempo de jejum inicial e pela disponibilidade de substrato para digestão e absorção; e ainda as características da dieta fornecida, cuja abrasividade afeta o turnover celular da membrana. Além disso, a ingestão de alimento, bem como as propriedades químicas dos nutrientes presentes no lúmen intestinal, é considerada um estímulo ao desenvolvimento da mucosa intestinal (Maiorka, 2001).

Acredita-se que o aumento no comprimento do intestino delgado e na área de superfície da mucosa com a idade é também uma estratégia para compensar a perda de atividade enzimática por célula ou por unidade de superfície da mucosa intestinal, que ocorre com o avanço da idade da ave. Em idades mais avançadas, as vilosidades se estendem para sustentar alta atividade total das enzimas em relação aos pequenos vilos de aves jovens (Iji et al., 2001b). 
Tabela 8 - Peso relativo (\% do peso vivo), comprimento $(\mathrm{cm})$ e densidade $(\mathrm{mg} / \mathrm{cm})$ do intestino delgado aos 1 , 4 e 7 dias de idade para frangos alimentados com diferentes fontes de carboidrato na dieta pré-inicial

Table 8 - Relative weight (\% of live weight), length $(\mathrm{cm})$ and density $(\mathrm{mg} / \mathrm{cm})$ of small intestine at 1, 4 and 7 days of age for broilers fed different carbohydrate sources in the diets

\begin{tabular}{|c|c|c|c|c|c|c|c|c|c|}
\hline & \multicolumn{3}{|c|}{$\begin{array}{l}\text { Peso relativo }(\% \mathrm{PV}) \\
\text { Relative weight }(\% L W)\end{array}$} & \multicolumn{3}{|c|}{$\begin{array}{l}\text { Comprimento }(\mathrm{cm}) \\
\text { Length }\end{array}$} & \multicolumn{3}{|c|}{$\begin{array}{c}\text { Densidade }(\mathrm{mg} / \mathrm{cm}) \\
\text { Density }\end{array}$} \\
\hline & $\begin{array}{l}1 \text { dia } \\
1 \text { day }\end{array}$ & $\begin{array}{l}4 \text { dias } \\
4 \text { days }\end{array}$ & $\begin{array}{l}7 \text { dias } \\
7 \text { days }\end{array}$ & $\begin{array}{l}1 \text { dia } \\
1 \text { day }\end{array}$ & $\begin{array}{l}4 \text { dias } \\
4 \text { days }\end{array}$ & $\begin{array}{l}7 \text { dias } \\
7 \text { days }\end{array}$ & $\begin{array}{l}1 \text { dia } \\
1 \text { day }\end{array}$ & $\begin{array}{l}4 \text { dias } \\
4 \text { days }\end{array}$ & $\begin{array}{l}7 \text { dias } \\
7 \text { days }\end{array}$ \\
\hline TES & 2,96 & $4,46 \mathrm{ab}$ & 4,54 & 53,00 & 80,00 & 96,50 & 32,50 & 60,00 & 82,50 \\
\hline AMI & 3,16 & $5,12 \mathrm{a}$ & 4,61 & 50,25 & 80,63 & 93,13 & 35,00 & 70,00 & 87,50 \\
\hline AMA & 3,07 & $4,52 \mathrm{ab}$ & 4,53 & 52,25 & 78,25 & 96,50 & 35,00 & 57,50 & 87,50 \\
\hline GLI & 3,11 & $4,20 \mathrm{~b}$ & 4,72 & 51,38 & 76,38 & 99,38 & 35,00 & 57,50 & 90,00 \\
\hline SAC & 3,80 & $4,45 \mathrm{ab}$ & 4,81 & 51,13 & 80,63 & 99,50 & 40,00 & 62,50 & 87,50 \\
\hline CV(\%) & 19,83 & 9,82 & 11,72 & 6,91 & 5,14 & 7,59 & 17,44 & 12,06 & 9,84 \\
\hline
\end{tabular}

a, b Médias seguidas de diferentes letras na coluna diferem entre si pelo teste Tukey $(P<0,10)$ (Average values followed by different letters in the same column differ $[P<$.10] by Tukey test).

Tabela 9 - Valores médios de ganho de peso (GP), consumo de ração (CR), conversão alimentar (CA), uniformidade (UNIF) e viabilidade criatória (VIAB) de frangos de corte de 8 a 21 dias de idade alimentados com diferentes fontes de carboidrato na fase pré-inicial

Table 9 - Average values of weight gain (GP), feed intake (CR), feed conversion (CA), uniformity (UNIF) and viability (VIAB) of the broilers from 8 to 21 days fed different carbohydrate sources from day 1 to 7

\begin{tabular}{lccccc}
\hline & $\begin{array}{c}\text { GP } \\
\text { (g/ave) } \\
(\text { g/bird })\end{array}$ & $\begin{array}{c}\text { CR } \\
\text { (g/ave) } \\
\text { (g/bird) }\end{array}$ & CA & $\begin{array}{c}\text { UNIF } \\
\text { dias (21 days) }\end{array}$ & $\begin{array}{c}\text { VIAB a 21 dias(8 to 21 days) } \\
(\%)\end{array}$ \\
\cline { 2 - 6 } TES & 611,3 & 877,6 & 1,41 & 11,28 & 99,69 \\
AMI & 614,4 & 869,6 & 1,40 & 12,02 & 100 \\
AMA & 638,3 & 934,6 & 1,47 & 7,72 & 100 \\
GL & 626,6 & 905,2 & 1,46 & 9,33 & 99,63 \\
SAC & 604,2 & 893,9 & 1,43 & 12,63 & 100 \\
CV(\%) & 4,56 & 4,32 & 3,61 & 41,91 & 0,44 \\
\hline
\end{tabular}

${ }^{1}$ Média do coeficiente de variação dos pesos individuais das aves de cada parcela dos diferentes tratamentos (Average value of coefficient of variation of individual live weight of birds of each pen of different treatments)

$\mathrm{CV}(\%)=$ coeficiente de variação (coefficient of variation).

Os valores médios dos tratamentos para as variáveis de desempenho, uniformidade e viabilidade criatória na fase de 8 a 21 dias de idade são apresentados na Tabela 9, não sendo observado efeito significativo $(\mathrm{P}>0,05)$ dos tratamentos.

O peso vivo das aves aos 21 dias foi 822, 816, 795 e $787 \mathrm{~g}$ para os tratamentos AMA, GLI, AMI e SAC, respectivamente. Todos estes valores foram numericamente superiores ao das aves do tratamento TES $(785 \mathrm{~g})$ na mesma idade, evidenciando efeito benéfico das fontes de carboidrato, sobretudo AMA e GLI.

Algumas estratégias devem ser estudadas para melhor aproveitamento dos efeitos benéficos dos carboidratos na fase pré-inicial, assim como a avalia- ção em condições práticas sobre piso e também a associação desses ingredientes com fontes de proteína de melhor qualidade.

\section{Conclusões}

Os valores da energia metabolizável aparente corrigida pelo balanço de nitrogênio determinado para frangos de corte na fase pré-inicial foram 3.269, 3.690, 3.427 e $3.524 \mathrm{kcal} / \mathrm{kg}$ (na matéria natural) para o amido de milho, amido de mandioca, glicose e sacarose, respectivamente. Quando se utilizaram estes valores energéticos para formular dietas isoenergéticas, não houve efeito dos carboidratos sobre o desempenho das aves e o desenvolvimento do trato gastrintestinal. 


\section{Agradecimento}

À Fundação de Amparo a Pesquisa do Estado de São Paulo (FAPESP), pelo suporte financeiro.

\section{Literatura Citada}

AKIBA, Y.; MURAKAMI, H. Partioning of energy and protein during early growth of broiler chicks and contribuition of vitteline residues. In: WORLD POULTRY SCIENCE CONFERENCE, 1995, Antalia, Turkey. Proceedings... Antalia: World Poultry Science Symposium, 1995. p.46-52.

BARANYIOVÁ, E.; HOLMAN, J. Morphological changes in the intestinal wall in fed and fasted chickens in the first week after hatching. Acta Veterinaria, v.45, p.151-158, 1976.

BATAL, A.B.; PARSONS, C.M. Utilization of different soy products as affected by age in chicks. Poultry Science, v. 82, p.454-462, 2003.

CHOTINSKY, D.; TONCHEVA, E.; PROFIROV, Y. Development of dissacharidases activity in the small intestine of broiler chickens. British Poultry Science, v.42, p.389-393, 2001.

CORLESS, A.B.; SELL, J.L. The effects of delayed acess to feed and water on the physical and functional development of the digestive system of young turkeys. Poultry Science, v.78, p.1158-1169, 1999.

DIBNER, J. Nutritional requirements of young poultry. In: ARKANSAS NUTRITONCONFERENCE, 1996, Fayetteville. Proceedings... Fayetteville: Arkansas Poultry Federation, 1996. p.15-27.

DONALDSON, W.E.; CHRISTENSEN, V.L. Dietary carbohydrate level and glucose metabolism in turkey poults. Comparative Biochemistry and Physiology, v.98, p. $347-350,1991$.

GUYTON, A.C. Tratado de fisiologia médica. 8.ed. Rio de Janeiro: Guanabara Koogan, 1992. 864p.

IJI, P.A.; SAKI, A.; TIVEY, D.R. Body and intestinal growth of broiler chicks on a commercial starter diet. 1. Intestinal weight and mucosal development. British Poultry Science, v.42, p.505-513, 2001a.

IJI, P.A.; SAKI, A.; TIVEY, D.R. Body and intestinal growth of broiler chicks on a commercial starter diet. 2. Development and characteristics of intestinal enzymes. British Poultry Science, v.42, p.514-522, 2001 b.

KATANBAF, M.N.; DUNNINGTON, E.A.; SIEGEL, P.B. Allomorphic relationships from hatching to 56 days in parental lines and $F_{1}$ crosses of chickens selected 27 generations for high or low body weight. Growth, Development and Aging, v.52, p.11-22, 1988.

LEVIN, R.J. Digestion and absorption of carbohydrate from embryo to adult. In: BOORMAN, K.N.; FREEMAN, B.M. (Eds.) Digestion in the fowl. Edinburgh: British Poultry Science Ltda, 1976. p.63-116.

MAIORKA, A. Adaptações digestivas pós-eclosão. In: CONFERÊNCIA APINCO'2001 DE CIÊNCIA E TECNOLOGIA AVÍCOLA - SIMPÓSIO INTERNACIONAL SOBRE MANEJO PRÉ E PÓS-ECLOSÃO, 2001, Campinas. Anais... Campinas: Fundação Apinco de Ciência e Tecnologia Avícolas, 2001. p.141-152.

MATEOS, G.G.; SELL, J.L. Influence of carbohydrate and supplemental fat source on the metabolizable energy of the diet. Poultry Science, v.59, p.2129-2135, 1980.
MATTERSON, L.D.; POTTER, L.M.; STUTZ, N.W. et al. The metabolizable energy of feed ingredients for chickens. Research Report, v.7, p.3-11, 1965.

MENTEN, J.F.M.; LONGO, F.A.; PEDROSO, A.A. et al. Valores de energia metabolizável de milho e farelo de soja para frangos de corte na fase pré-inicial. In: REUNIÃO ANUAL DA SOCIEDADE BRASILEIRA DE ZOOTECNIA, 39., 2002, Recife. Anais... Recife: SBZ, 2002. CD-ROM. Nutrição de Não-Ruminantes

MORAN JR., E.T. Digestion and absortion of carbohydrates in fowl and events through perinatal development. Journal of Nutrition, v.115, p.665-674, 1985.

MOZDZIAK, P.E.; WALSH, T.J.; McCOY, D.W. The effect of early posthatch nutrition on satellite cell mitotic activity. Poultry Science, v.81, p.1703-1708, 2002.

MURAKAMI, H.; AKIBA, Y.; HORIGUCHI, M. Growth and utilization of nutrients in newly-hatched chick with or without removal of residual yolk. Growth, Development and Aging, v.56, p.75-84, 1992.

NITSAN, Z.; TURRO-VINCENT, I.; LIU, G. et al. Intubation of weight-select chicks with soybean oil or residual yolk: effect early growth and development. Poultry Science, v.74, p.925-936, 1995.

NOY, Y.; SKLAN, D. Digestion and absorption in the young chick. Poultry Science, v.74, p.366-373, 1995.

NOY, Y.; GEYRA, A.; SKLAN, D. The effect of early feeding on growth and small intestinal development in the posthatch poult. Poultry Science, v.80, p.912-919, 2001.

NATIONAL RESEARCH COUNCIL - NRC. Nutrients requirements of poultry. Washington, D.C.: National Academiy Press, 1994, 155p.

ROSTAGNO, H.S.; ALBINO, L.F.T.; DONZELE, J.L. et al. Tabelas brasileiras para aves e suínos: composição de alimentos e exigências nutricionais. Viçosa, MG: Universidade Federal de Viçosa, 2000. 141p.

STATISTICAL ANAYSES SYSTEM - SAS. SAS user's guide. Version 6.11. Cary: 1996. 956p.

SELL, J.; ANGEL, C.; PIQUER, F. et al. Development patterns of selected characteristics of the gastrointestinal tract of young turkeys. Poultry Science, v.70, p.1200-1205, 1991.

SIDDONS, R.C. Effect of diet on disaccharidase activity in the chick. British Journal of Nutrition, v.27, p.343-352, 1972.

SILVA, D.J. Análise de alimentos (Métodos químicos e biológicos). Viçosa, MG: Universidade Federal de Viçosa, 1990, 165p.

SORBARA, J.O.B. Efeito de diferentes carboidratos na ração pré-inicial de frangos de corte sobre o desempenho e a alometria dos órgãos. Piracicaba: Escola Superior de Agricultura Luiz de Queiroz, 2003. 60p. Dissertação (Mestrado em Agronomia) - Escola Superior de Agricultura Luiz de Queiroz, 2003.

SULISTYANTO, B.; AKIBA, Y.; SATO, K. Energy utilization of carbohydrate, fat and protein sources in newly hatched chicks. British Poultry Science, v.40, p.653-659, 1999.

UNI, Z.; GANOT, S.; SKLAN, D. Post-hatch development of mucosal function in the broiler small intestine. Poultry Science, v.77, p.75-82, 1998.

UNI, Z.; NOY, Y.; SKLAN, D. Post-hatch changes in morphology and function of the small intestine in heavy and light strain chicks. Poultry Science, v.74, p.1622-1629, 1995.

Recebido em: 04/11/03 Aceito em: 17/09/04

R. Bras. Zootec., v.34, n.1, p.123-133, 2005 